\title{
ÍNDICE DE ALIMENTACIÓN SALUDABLE, INGESTA DE AGUA Y CALIDAD DEL SUEÑO EN ATLETAS DE ALTO RENDIMIENTO DE UNA UNIVERSIDAD PÚBLICA
}

\author{
HEALTHY EATING INDEX, WATER INTAKE AND SLEEP QUALITY IN HIGH PERFORMANCE ATHLETES FROM
} A PUBLIC UNIVERSITY.

\author{
Núñez-Rocha Georgina Mayela ${ }^{1}$, Martínez-Hernández Rocío ${ }^{1}$, Cañamar-Ramírez Mayra², Ávila-Ortiz María \\ Natividad $^{1}$, Pérez García José Alberto ${ }^{2}$, Guevara-Valtier Milton Carlos ${ }^{3}$, Hernández Ruiz Karina Janett ${ }^{1}$. \\ Universidad Autónoma de Nuevo León: 1 Facultad de Salud Pública y Nutrición, 2 Dirección de Deportes, 3 Facultad de \\ Enfermería. México.
}

\begin{abstract}
RESUMEN
Introducción. Los atletas de alto rendimiento (AAR) deben tener una alimentación saludable, ingesta adecuada de agua y buena calidad del sueño para mejorar su rendimiento físico. Objetivo: Determinar el índice de alimentación saludable, la ingesta de agua en 24 horas y la calidad del sueño, en AAR de una universidad pública. Material y Método: Diseño transversal descriptivo, participaron N= 769 AAR. Se aplicó una encuesta digital previa autorización y firma de consentimiento informado. Contenía variables sociodemográficas y tipo de deporte. Se estableció el Índice de Alimentación Saludable mediante frecuencia de consumo alimentario, se midió ingesta de agua en 24 horas y calidad del sueño. Se aplicaron promedios, desviación estándar, frecuencias y porcentajes. Resultados: El 57.7\% eran mujeres, edad de 19.8 2 ,6 años. Predominaron los deportes de pelota. El $25 \%$ mostró una alimentación saludable; el consumo de agua fue de $1.825 \pm .828$ litros en 24 horas; el $48 \%$ de las mujeres y $36.8 \%$ de los hombres tuvieron ingesta adecuada de agua. El $24,4 \%$ presentó buena calidad de sueño. Conclusiones: La prevalencia de alimentación saludable, ingesta adecuada de agua y una buena calidad del sueño es baja. Palabras Clave: Atletas de alto rendimiento, alimentación saludable, ingesta de agua, calidad del sueño.
\end{abstract}

\section{ABSTRACT}

Introduction: Athletes should have a healthy diet, adequate water intake and good sleep quality to improve their physical performance. Objective: To determine the index of healthy eating, water intake and sleep quality in high performance athletes. Material and method: Cross-sectional descriptive design, $\mathrm{N}=\mathbf{7 6 9}$ high performance athletes participated. After authorization and signature of informed consent, an online survey was applied. It contained sociodemographic variables and type of sport. The Healthy Eating Index was established through frequency of food consumption, water intake in 24 hours and sleep quality. Averages, standard deviation, frequencies and percentages were applied. Results: $57.7 \%$ were women, age $19.8 \pm 2.6$ years. Ball sports were predominant. $25 \%$ showed a healthy diet; water consumption of $1.825 \pm .828$ liters in 24 hours; $48 \%$ of women and $36.8 \%$ of men had adequate water intake. A good quality of sleep was observed in $24.4 \%$. Conclusion: The prevalence of healthy eating, adequate water intake and good sleep quality is low. It is important to design strategies to improve these habits and optimize physical performance, quality of life and health.

Key words: High performance athletes, healthy eating, water intake, sleep quality.

Correspondencia: Georgina Mayela Núñez Rocha.georgina.nunezr@uanl.mx

Recibido: 07 de julio 2021, aceptado: 08 de septiembre 2021

(C) Autor2021

(c) (i)

DOI: https://doi.org/10.29105/respyn20.4-3

Citation: Núñez-Rocha G.M., Martínez - Hernández R., Cañamar-Ramírez M., Ávila-Ortiz M.N., Pérez García J.A., Guevara-Valtier M.C., Hernández Ruiz K.J. (2021) Índice de alimentación saludable, ingesta de agua y calidad del sueño en atletas de alto rendimiento de una universidad pública. Revista Salud Pública y Nutrición, 20 (4), 22-30. 


\section{Artículo Original}

\section{Introducción}

De acuerdo a la Ley General de Cultura Física y el Deporte en México (2013): El atleta de alto rendimiento (AAR) se define como "aquel que practica la disciplina deportiva con altas exigencias técnicas y científicas de preparación y entrenamiento, que le permiten participar en preselecciones y selecciones nacionales que representan al país en competencias y pruebas oficiales de carácter internacional" (Ley General de Cultura Física, México, 2013).

En AAR, la alimentación saludable, la adecuada ingesta de agua y calidad de sueño son necesarias para preservar la salud y tener una buena calidad de vida, además, mejoran el rendimiento físico antes, durante y después de los entrenamientos de rutina. Publicaciones señalan que estas acciones están directamente relacionadas con mejores resultados en sus competencias deportivas (Kotarska, Nowak, Szark \& Nowak, 2019; Parrón, Nestares \& De Teresa, 2015; Duarte \& Anderson, 2013). En este grupo poblacional, una alimentación saludable hace referencia a la incorporación de diferentes grupos de alimentos como: cereales, frutas, verduras, lácteos, carnes, y legumbres en las porciones y frecuencias de consumo recomendadas por organismos e instituciones de salud. (American Dietetic Association, 2019; Pelly \& Thurecht, 2019; BertiZanella, Maciel-August, Donner-Alves, Matte, Guerini de Souza, 2018). Por otro lado, una alimentación poco saludable tiene impacto directo en la pérdida de masa muscular, pérdida o fracaso para ganar densidad ósea, elevado riesgo de sufrir lesiones, desórdenes alimentarios, osteoporosis y amenorrea en el caso de mujeres y niveles prolongados de cansancio (American Dietetic Association, 2029; Pelly et al., 2019; Berti-Zanella et al., 2018; Belval, Hosokawa, Casa, Adams, Armstrong, Baker et al., 2019; Taylor, Chrismas, Dascombe, Chamari, Fowler, 2016). La alimentación de los AAR se puede ver afectada por factores externos como la comercialización de alimentos ultra procesados, viajes recurrentes que cambian su estilo de alimentación, y limitación en la misma por el grupo de alimentos existentes en los comedores. Aunado a lo anterior, se conoce que este grupo de población suele consumir grandes cantidades de alimentos cuya calidad nutritiva es deficiente, ingieren bebidas azucaradas en forma excesiva y la ingesta de agua y vegetales es baja lo que conlleva al consumo insuficiente de micronutrientes y fibra (Massarani, Citelli, Canella \& Koury,2019; Trakman, Forsyth, Hoye, Belski, 2019; Mancine, Kennedy, Stephan, Ley, 2021).

En relación a la ingesta de agua, diferentes instituciones como la Federación Española de Medicina Deportiva (Belval et al., 2019) señala la importancia de la ingesta de agua en AAR cuya cantidad mínima es de 3 litros de agua natural por día. Este requerimiento radica en la demanda física que realiza este grupo de personas para cumplir con las expectativas de los entrenamientos con respecto al deporte que practican; en este sentido, las demandas físicas y fisiológicas son representadas por la composición muscular, el proceso de termorregulación, la tasa de sudoración, rutinas de ejercicio y otros factores que los hacen vulnerables a un desequilibrio hídrico por una ingesta insuficiente de líquido particularmente de agua (Belval et al., 2019; Olzinski, Beaumont, Toledo, Yudell, Johnston \& Wardenaar, 2019;:Iglesias,Villarino, Martínez, Cabrerizo, Gallardo, Lorenzo, et al. 2011).Una deshidratación repercute directamente en los músculos y estructuras tendinoligamentosas, ya que produce rigidez y reduce los procesos fisiológicos del organismo, provocando una disminución en su rendimiento físico y aumenta el riesgo de sufrir desmayos, agotamiento por calor y en casos extremos la muerte (Olzinski et al, 2019; Barbero, Castagna \& Granda, 2006). Al respecto, estudios de investigación señalan que una pérdida de peso del $2 \%$ por deshidratación, provoca una disminución del 5 al $10 \%$ del rendimiento físico (Urdampilleta, Martínez, Julia \& Álvarez, 2013; Observatorio de Hidratación y Salud, 2007) finalmente y no menos importante la deshidratación, incluso en niveles muy bajos puede afectar también la calidad del sueño (Belval, et al, 2019; Taylor, 2016)

La buena calidad del sueño en AAR, es imprescindible no solo por los beneficios que ya se conocen, sino porque es el medio más efectivo y natural para favorecer y acelerar los procesos de recuperación muscular. Una mala calidad del sueño puede propiciar altos niveles de cansancio diurnos, afectando directamente la calidad y la adherencia al entrenamiento y aumenta el riesgo de sufrir lesiones; además, reduce el funcionamiento físico y cognitivo. Existe evidencia científica de la relación directa entre mala calidad del sueño y perder durante una 


\section{Artículo Original}

competencia deportiva; asimismo, entre la duración del sueño y el rendimiento en dicha competencia (Gupta, Morgan \& Gilchrist, 2017; Kölling, Duffield, Erlacher, Venter \& Halson, 2019) .Entre los factores relacionados con la degradación en la calidad de sueño de los AAR se encuentran las demandas de entrenamiento (frecuencia, intensidad y volumen),mayor excitación antes de las competencias, uso de dispositivos electrónicos antes de acostarse y la demanda de viajes debido a las competencias deportivas (Gupta et al 2017; Kölling et al, 2019; Mata-Ordoñez, CarreraBastos,Domínguez,Sánchez,Oliver,2018;Poussel,La ure,GenestFronzaroli,Renaud,Favre.etal.2014).

La necesidad de continuar con el estudio de estas variables es porque se podrían asumir comportamientos saludables en este grupo y no es correcto considerar de antemano que los AAR tienen estilos de vida saludables. Publicaciones refieren que 12 a $32 \%$ de AAR no lleva una alimentación saludable, que 43 a $80.2 \%$ no incluye en su dieta alimentos de consumo diario como son las frutas o verduras y $17 \%$ tiene una mala o muy mala alimentación. Por otro lado, se ha encontrado que 40 a $48.6 \%$ de los atletas ingiere menos de 1.500 litros diarios de agua, incluso hay evidencia de que un alto porcentaje $(67 \%)$ no tiene una hidratación adecuada posterior al ejercicio (Belval et al, 2019; Taylor et al, 2016; Parrón et al, 2015). En relación a la calidad del sueño se ha reportado que 30 a $65.8 \%$, tiene una mala calidad de sueño (Belval et al., 2019; Taylor et al, 2016; Mancine et al., 2021; Leduc, Tee, Weakley, Ramírez, Jones, 2019). Desde esta perspectiva, el presente estudio tiene por objetivo determinar el índice de alimentación saludable, la ingesta de agua en 24 horas y la calidad del sueño en AAR de una universidad pública.

\section{Material y Método}

Se llevó a cabo un estudio transversal descriptivo, en una población de AAR de diferentes tipos de deporte de una Universidad pública. Se incluyó el total (censo) de atletas que aceptaron participar en el estudio $(\mathrm{N}=769)$ y que firmaron la carta de consentimiento informado. Se excluyeron aquellos con lesiones en los últimos tres meses y los participantes con encuestas incompletas $(\mathrm{N}=8)$. Se determinó un tamaño mínimo de muestra bajo la hipótesis que menos de $20 \%$ de los AAR tenía una alimentación saludable con el $95 \%$ de nivel confianza y un nivel de significancia de $5 \%$ dio un total de $n=219$ participantes. El muestreo fue no probabilístico por conveniencia considerando al total de AAR.

Debido a la emergencia sanitaria actual, se diseñó una encuesta digital la cual incluía variables sobre tipo de deporte: resistencia y fuerza rápida (atletismo, escalada y triatlón y levantamiento de pesas); coordinación y arte competitivo (animación, gimnasia aeróbica, natación, wáter polo, tiro con arco y ajedrez); combate (box, esgrima, judo, karate y tae kwon do); juegos de pelota (football americano, basketball, football rápido, football soccer, tenis, tenis de mesa, voleibol, voleibol de playa, handball, hockey de pasto, softball y tochito). Variables sociodemográficas como edad en años cumplidos; sexo, facultad a la que pertenecían y semestre que cursaban; lugar de origen: Nuevo León /otro estado de la república/otro país; ocupación: trabaja/no trabaja; estado civil: con pareja/sin pareja; con quien vive: con sus padres/compañero de cuarto/ con pareja/alojamiento de la Universidad y si utilizaban el Servicio de Alimentación y Nutrición Deportiva de la Universidad antes de la emergencia sanitaria.

Para establecer el Índice de Alimentación Saludable (IAS) se aplicó la encuesta de Frecuencia de Consumo Alimentario (FCA) compuesta por 9 grupos de alimentos (cereales, frutas, verduras, lácteos, proteínas, legumbres, embutidos, repostería y aceites o grasas) de acuerdo a la recomendación de la Sociedad Española de Nutrición Comunitaria los primeros cuatro grupos (cereales, verduras y hortalizas, frutas y leche y derivados) son de consumo diario; si se consumían diariamente se daba puntuación de 10 , si se consumían 3 o más veces, pero no a diario 7.5, si se consumían 1 o 2 veces a la semana 5 , menos de una vez a la semana 2.5 y nunca o casi nunca la puntuación era de 0 . Los dos siguientes grupos (carnes y legumbres) son de consumo semanal; si se consumían 1 o 2 veces a la semana se daba puntuación de 10 , si se consumía 3 o más veces a la semana, pero no diario 7.5 , si se consumía menos de una vez a la semana 5 , consumo diario 2.5, nunca o casi nunca la puntuación sería 0 . Finalmente, los tres últimos grupos (embutidos, dulces y refrescos con azúcar, aceites o grasas) son de consumo ocasional; si nunca o casi nunca se consumían, la puntuación era de 10 , menos de una vez a la semana7.5, 1 o 2 veces a la semana 5,3 o 
más veces a la semana 2.5 y si se consumían diariamente la puntuación era 0 . En cuanto a la variedad, se otorgaron 2 puntos si cumplía cada una de las recomendaciones diarias para cada grupo de alimentos y 1 punto si cumplía cada una de las recomendaciones semanales. La sumatoria de las 10 variables da un puntaje máximo de 100 y los puntos de corte son de acuerdo con el puntaje total: $>80$ puntos=alimentación saludable, $\quad 50$ a 80 puntos $=$ necesita cambios y $<50$ puntos $=$ alimentación poco saludable. (Berti-Zanella et al., 2018; Norte \& Ortiz, 2011; Krebs, Pannucci, Subar, Kirkpatrick, Lerman, Tooze et al.,2018).

En relación a la ingesta de agua, ésta se estableció mediante auto reporte de la cantidad en promedio de ingesta de agua en litros cada 24 horas y se categorizó de acuerdo al sexo en adecuada, moderada y deficiente, siguiendo la norma establecida por la Federación Española de Medicina Deportiva (Belval et al., 2019) que recomienda para mujeres una ingesta diaria de aproximadamente de 2 a 2.5 litros de agua y hombres de 2.5 a 3 litros. (Urdampilleta et al., 2013; Iglesias et al., 2011; Observatorio de Hidratación y Salud, 2007).

La calidad del sueño se evaluó mediante el instrumento de Pittsburgh Sleep Quality Index (PSQI) (Buysse, Reynolds, Monk, Berman, Kupfer, 1989; Leduc et al., 2019). El cual está compuesto por 7 dimensiones cuya sumatoria es igual a 21, los puntos de corte son: $>5$ que indica una mala calidad del sueño y $<5$ que sugiere una buena calidad del sueño.

Previa gestión en reunión con entrenadores de las diferentes disciplinas deportivas, se les envió a los atletas en el mes de enero 2021 un enlace electrónico de la encuesta diseñada en Google Drive junto con el consentimiento informado para que lo firmaran aquellos que desearan participar; posteriormente, respondieron la encuesta y enviaron sus respuestas. Dicha encuesta tiene una duración de 15 a 20 minutos aproximadamente. Se excluyeron $\mathrm{N}=8$ encuestas incompletas. El plan de análisis incluyó estadística descriptiva: promedio y desviación estándar de las variables numéricas y frecuencias y porcentajes de las variables categóricas. Se utilizó el programa IBM Statistical Package for the Social Sciences (SPSS) versión 21.
El estudio fue aprobado por el Comité de Ética e Investigación de la Facultad de Salud Pública y Nutrición con número de registro 20-FaSPyN-SA17.TP. Se guardó anonimato y confidencialidad de los datos

\section{Resultados}

Características generales y sociodemográficas. Participaron $\mathrm{N}=769$ AAR de diferentes tipos de deporte. Predominaron los atletas que practicaban juegos de pelota (figura 1).

Figura 1

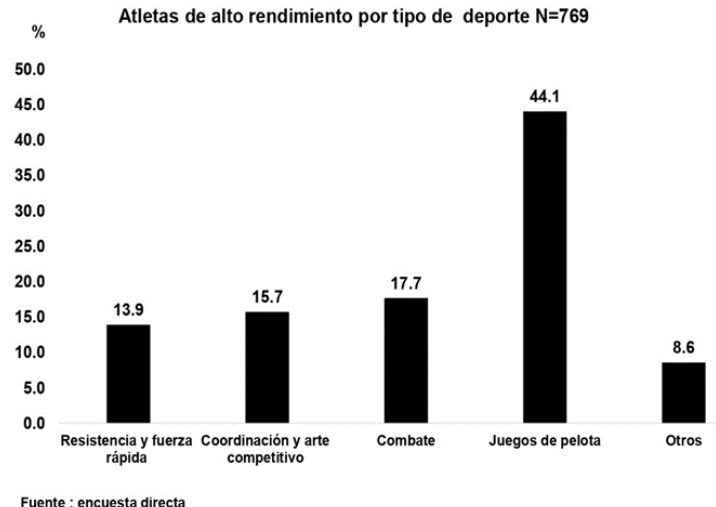

El $57.7 \%$ eran mujeres, el promedio de edad fue de $19.8 \pm 2.6$ años, $64.6 \%$ era de Nuevo León, $33.2 \%$ trabajaba, $98.8 \%$ no tenía pareja, $84.3 \%$ vivía con sus padres, $22.1 \%$ del total de participantes pertenecía a la Facultad de Ingeniería Mecánica y Eléctrica y 21.7 $\%$ cursaba tercer semestre de la licenciatura. El $35.4 \%$ de los atletas utilizaba el servicio de alimentación deportiva antes de la emergencia sanitaria (tabla 1) 
(n)

Tabla 1. Carácterísticas generales y demográficas de los atletas

\begin{tabular}{llrr}
\hline Variables & & $\mathrm{n}$ & $\%$ \\
\hline \multirow{2}{*}{ Sexo } & Mujer & 444 & 57.7 \\
& Hombre & 325 & 42.3 \\
& Ingeniería mecánica y eléctrica & 169 & 22.1 \\
& Organización deportiva & 141 & 18.3 \\
Facultad & Contaduría pública y & 137 & 17.8 \\
& administración & 86 & 11.2 \\
& Derecho y criminología & 236 & 30.6 \\
& Otras Facultades & & \\
& Primer semestre & 142 & 18.3 \\
Semestres & Tercer semestre & 167 & 21.7 \\
& Quinto semestre & 131 & 17 \\
& Otros semestres & 329 & 43 \\
Lugar origen & & & \\
& Monterrey & 497 & 64.6 \\
Trabajo & Otro estado o país & 272 & 35.4 \\
& Si & 255 & 33.2 \\
& No & 514 & 66.8 \\
Estado civil & Soltero & 756 & 98.3 \\
& Unión libre & 13 & 1.7 \\
Con quien vive & Con padres & 648 & 84.3 \\
& Otras personas & 39 & 10.6 \\
& Solo & 5.1 \\
\hline
\end{tabular}

Utilizaban el Servicio

de Alimentación

Deportiva de la

universidad antes de la
emergencia sanitaria.

Fuente: encuesta directa

Índice de alimentación saludable: Se observó que $70 \%$ de los atletas necesita cambios en su alimentación (figura 2).

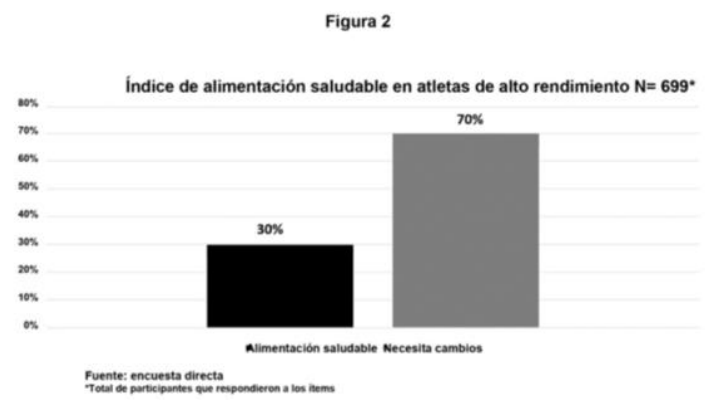

Ingesta de agua. El promedio de consumo de agua de los atletas fue de $1.825 \pm .828$ litros al día y la ingesta insuficiente de agua al día por sexo resultó ser mayor en los hombres (figura 3).
Figura 3

Ingesta de agua por sexo en atletas de alto rendimiento $\mathrm{N}=767^{*}$
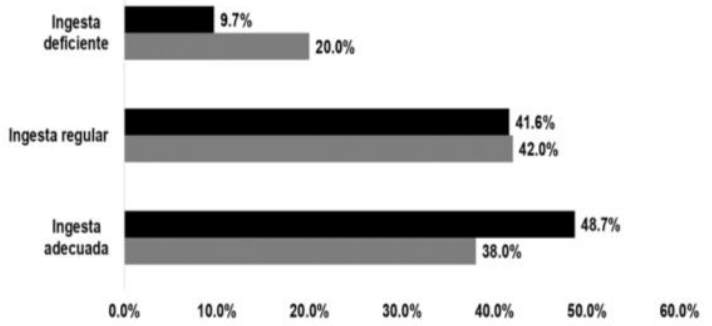

- Mujeres (n=444) $\quad$ Hombres (n=323)

Fuente : encuesta directa
Total de participantes que respondieron a los items

Calidad del sueño. El 73.0\% de los atletas presentó mala calidad del sueño como se observa en la tabla 2.

\begin{tabular}{l}
\multicolumn{3}{l}{ Tabla 2. Calidad del sueño en atletas } \\
\begin{tabular}{lcr}
\hline Calidad del sueño & $\mathrm{n}$ & $\%$ \\
\hline Buena & 208 & 27 \\
Mala & 561 & 73 \\
Total & 769 & 100 \\
\hline Fuente: encuesta directa & \\
$\mathrm{n}=769$
\end{tabular}
\end{tabular}

Mientras que, en la evaluación de la calidad del sueño por sexo, las mujeres presentaron mayor prevalencia de mala calidad del sueño frente a los hombres (figura 4).

Figura 4

Calidad del sueño en atletas de alto rendimiento por sexo $N=749^{*}$

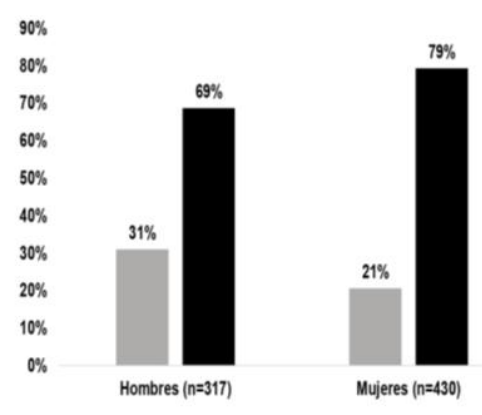

"Buena Calidad del Sueño - Mala Calidad del Sueño

Fuente : encuesta directa
Total de participantes que respondieron a los items 


\section{Artículo Original}

\section{Discusión}

La alimentación, la ingesta de agua y la calidad del sueño son el primer paso para contar con un panorama general del estado de salud de los AAR, es imprescindible identificar estas prácticas de manera cotidiana ya que son la brújula para el diseño de estrategias individualizadas y efectivas que optimicen su rendimiento deportivo.

Como se observó en el presente estudio, la mayoría de los atletas necesita cambios en su alimentación de acuerdo al índice de alimentación saludable que se estableció y a lo recomendado por las diferentes sociedades de nutrición clínica (Norte A \& Ortiz R. 2011), y deportiva (Berti-Zanella et al, 2019; American Dietetic Association, 2009); estos hallazgos concuerdan con lo encontrado por BertiZanella y colaboradores (2019) donde se estableció que $72,7 \%$ de los atletas presentaba una alimentación poco saludable.

Otro estudio estableció el consumo de alimentos en atletas y se observó que solo el $12 \%$ de ellos cumplía con las recomendaciones de la pirámide de alimentación saludable propuesto por la Organización de la Naciones Unidas para la Alimentación y Agricultura (FAO) (Parrón-Sevilla., et al, 2015), lo mismo que en el presente estudio, los niveles bajos de alimentación saludable en los AAR puede explicarse por diversos factores como la falta de asesoría nutricional, falta de conocimientos con respecto a una alimentación saludable e incluso el confinamiento debido a la actual pandemia y la falta de competencias próximas, que involucra el máximo apego del AAR a una alimentación saludable.

En lo que respecta a la ingesta de agua, los hallazgos encontrados están por debajo de la ingesta media de las recomendaciones de la Federación Española de Medicina del Deporte (Belval, et al, 2019; Olzinski, et al, 2019; Iglesias, et al, 2011; Serra, et al. 2007), estos hallazgos coinciden con lo referido por ParrónSevilla y colaboradores (2015), donde miden la ingesta de agua diaria mediante el auto reporte en jugadores de pádel y se encontró que $48.6 \%$ de los atletas no ingería la cantidad mínima de 1500 mililitros de agua diarios recomendados. Por otro lado, en corredores de montaña, se comparó el consumo de carbohidratos, agua y sodio con la recomendación actual y se encontró que la ingesta media de agua era de $1591.67 \pm 630$ mililitros; los resultados de estos hallazgos se atribuyeron a la falta de conocimientos sobre la importancia de la hidratación y el impacto en el rendimiento físico cuando se mantiene hidratado durante todo el día (Jiménez-Alfageme, Aguirre, Mielgo-Ayuso, \& Martínez, 2021).

En relación a la calidad del sueño, los hallazgos muestran que la mayoría de los AAR presentan mala calidad del sueño lo cual concuerda con los resultados encontrados en otros estudios como el realizado por Leduc C., et al. (2019) donde determina la calidad, cantidad y variabilidad intra-individual del sueño en estudiantes y estudiantes-atletas de universidades del Reino Unido. Se midió mediante el instrumento de PSQI y se encontró que el $65 \%$ de los participantes presentaba mala calidad del sueño. Asimismo, un estudio realizado en Irlanda y el Reino Unido en atletas de sub élite y élite, estableció la calidad, cantidad y tiempo de sueño mediante el instrumento PSQI reportó que 64\% de los atletas presentaba mala calidad del sueño (Doherty, Madigan, Nevill, Warrington, \& Ellis, 2021). Los participantes expresaron que esta situación podría deberse a la situación de confinamiento, altas cargas de tarea, ansiedad y hambre nocturna entre otras.

Es conveniente mencionar que el presente estudio se realizó en el total de AAR de una universidad pública y de ambos sexos y aunque se han realizado estudios de estas variables en atletas, la mayoría las mide de manera aislada, no se ha publicado alguno que incluya estas tres de variables del llamado "entrenamiento invisible" (Rosero, 2018; Parrón, 2015). Por otro lado, es un estudio transversal que no permite por el momento hacer inferencias sino solo mostrar la situación actual.

\section{Conclusiones}

La mayoría de los atletas necesita cambios en su alimentación de acuerdo al índice de alimentación saludable, el promedio de ingesta de agua está por debajo de las recomendaciones y los hombres presentaron mayor prevalencia de inadecuada ingesta de agua en comparación con las mujeres y la mayoría de los atletas presenta mala calidad del sueño. Desde esta perspectiva, el presente estudio ilustra un panorama general de la situación que viven los AAR en relación a los hábitos saludables que son imprescindibles para conservar su salud y se relacionan estrechamente con el rendimiento físico 
antes, durante y después de sus entrenamientos y competencias deportivas. Por tanto, con estos resultados se tienen elementos sensibles para diseñar una intervención dirigida a este grupo de atletas con el fin de mantener y mejorar su salud en esta situación de emergencia sanitaria que los ha mantenido en confinamiento. Lo anterior es de gran trascendencia para mantener una supervisión y evaluación constante, estrecha e individualizada en donde se considere incluir la recopilación de datos de actividad física, datos antropométricos, bioquímicos, clínicos, ambientales y la ingesta de alimentos con porciones para el cálculo de la ingesta de macronutrientes de acuerdo a las necesidades individualizadas de cada atleta. Además, es importante diseñar y aplicar estrategias efectivas para incrementar la ingesta de agua y calidad del sueño para preservar la salud y optimizar su rendimiento físico.

\section{Agradecimientos}

A la Dirección de Deportes de la Universidad y a los entrenadores de los atletas de alto rendimiento participantes por su invaluable apoyo en la realización del presente estudio.

\section{Bibliografía}

American Dietetic Association. (2009). Position of the American Dietetic Association, Dietitians of Canada, and the American College of Sports Medicine: Nutrition and Athletic Performance. Journal of the American Dietetic Association, 109, (3) 509-27. DOI: 10.1249/MSS.0b013e31890eb86

Barbero, J., Castagna, C. \& Granda, J. (2006). Deshidratación y reposición hídrica en jugadores de fútbol sala: efectos de un programa de intervención sobre la pérdida de líquidos durante la competición. Motricidad. European Journal of Human Movement, 17,93-106. Disponible en: https://www.redalyc.org/pdf/2742/27422044200 7.pdf

Belval, L., Hosokawa Y., Casa, D., Adams, W., Armstrong, L., Baker, L., Burke, L., Cheuvront, S., Chiampas, G., González-Alonso, J., Huggins, R., Kavouras, S., Lee, E., McDermott, B., Miller, K., Schlader, Z., Sims, S., Stearns, R., Troyanos,
C and Wingo, J. (2019). Nutrients, 11, (7), 2-15. DOI: $10.3390 /$ nu 11071550

Berti-Zanella, P., Maciel-August, P., Donner-Alves, F., Matte, C. \& Guerini de Souza, C. (2018). Association of healthy eating index and oxidative stress in adolescent volleyball athletes and nonathletes. Nutrition, 60, 230-234 DOI: 10.1016/j.nut.2018.10.017

Buysse, DJ., Reynolds, CF., Monk, TH., Berman, SR. \& Kupfer, DJ. (1989). The Pittsburgh Sleep Quality Index: a new Instrument for psychiatric practice and research. Psychiatric Research, 28,193-213. DOI: $\quad$ 10.1016/01651781(89)90047-4

Cámara de Diputados del H. Congreso de la Unión. Ley General de Cultura Física y el Deporte. Disponible en: www.diputados.gob.mx/LeyesBiblio/pdf/LGCF D_190118.pdf

Doherty, R., Madigan, S.M., Nevill, A., Warrington, G., \& Ellis, J. G. (2021). The Sleep and Recovery Practices of Athletes. Nutrientes, 13(4), 1330. https://doi.org/10.3390/nu13041330.

Duarte, E. \& Anderson, G. (2013). Programa de autocuidado para el mejoramiento de la calidad de vida de atletas universitarios. Revista de Enfermería Actual de Costa Rica, 25,1-13. DOI: 10.15517/revenf. v0i25.11838

Gupta, L., Morgan, K. \& Gilchrist S. (2017). Does Elite Sport Degrade Sleep Quality? A Systematic Review. Sports Medicine, 47,1317-1333. DOI: 10.1007/s40279-016-0650-6

Iglesias, R., Villarino, A., Martínez, J., Martínez, J., Cabrerizo, L., Gargallo, M., Lorenzo, H., Quiles, J., Planas, M., Polanco, I., Romero de Ávila, D., Russolillo, J., Farré, R., Moreno, J., Riobó, P., Salvadó, S. (2011). Importancia del agua en la hidratación de la población española: documento FESNAD 2010. Nutrición Hospitalaria, 26, (1), 27-36. DOI:10.3305/nh.2011.26.1.5167

Jiménez-Alfageme, R., Aguirre López, L., MielgoAyuso, J., \& Martínez Sanz, J.M. (2021). Analysis of nutritional intake in trail runners 
during competition. Nutrición Hospitalaria, 38(2), 321-327. DOI.org/10.20960/nh.03388

Kölling, S., Duffield, R., Erlacher, D., Venter, R. \& Halson, S. (2019). Sleep-Related Issues for Recovery and Performance in Athletes. International Journal of Sports Physiology and Performance, $\quad 14, \quad$ (2),144-148. DOI.org/10.1123/ijspp.2017-0746

Kotarska, K., Nowak, L., Szark-Eckardt, M., \& Nowak, M. A. (2019). Intensity of Health Behaviors in People Who Practice Combat Sports and Martial Arts. International Journal Environmental Research and Public Health, 16, (14), 2463. DOI:10.3390/ijerph16142463

Krebs, S., Pannucci, T., Subar, A., Kirkpatrick, S., Lerman, J., Tooze, J., Willson, M. and Reeder, J. (2018). Update of the Healthy Eating Index: HEI2015. Journal of the Academy of Nutrition and Dietetics, 118, (9),1591-1602. DOI: 10.1016/j.jand.2018.05.021

Leduc, C., Tee, J., Weakley, J., Ramírez, C. \& Jones B. (2019). The Quality, Quantity, and Intraindividual Variability of Sleep Among Students and Student-Athletes. Salud Deportiva, 12, (1), 43-50. DOI:10.1177/1941738119887966

Lloret, M. (1989). El entrenamiento invisible. Apunts. Educación física y deportes, 1, (15), 1014. Disponible en: https://raco.cat/index.php/ApuntsEFD/article/vie w/382890

Mata-Ordoñez, F., Carrera-Bastos, P., Domínguez, R. \& Sánchez-Oliver, A.J. (2018). Importancia del sueño en el rendimiento y la salud del deportista. Revista de Educación, Motricidad e Investigación, 11,70-82. Disponible en: http://rabida.uhu.es/dspace/bitstream/handle/102 72/15796/Importancia.pdf?sequence $=2$

Mancine, R., Kennedy, S., Stephan, P. \& Ley A. (2020). Disordered Eating and Eating Disorders in Adolescent Athletes. Spartan Medical Research Journal, 4 (2), 1-5. DOI: 10.51894/001c.11595
Massarani, F., Citelli, M., Canella DS. \& Koury, J. (2019). Healthy eating promoting in a Brazilian sports-oriented school: a pilot study. Journal of Life and Environmental Sciences, 7, (7), 1-13. DOI: $10.7717 /$ peerj.7601

Norte A, Ortiz R. (2011). Calidad de la dieta española según el índice de alimentación saludable. Nutrición Hospitalaria, 26, 2, 330336. DOI:10.3305/nh.2011.26.2.4630

Olzinski, S., Beaumont, J., Toledo, M., Yudell, A., Johnston, C. \& Wardenaar, F. (2019). Hydration Status and Fluid Needs of Division I Female Collegiate Athletes Exercising Indoors and Outdoors. Sports, 7, (155),1-14. DOI: $10.3390 /$ sports 7070155

Parrón, E., Nestares, T. \& De Teresa, C. (2015). Valoración de los hábitos de vida saludables en jugadores de pádel. Annals of Sports Medicine, 8 , (4), 184-189. DOI: 10.1016/j.ramd.2015.08.002

Pelly, F. \& Thurecht, R. (2019). Evaluation of Athletes' Food Choices during Competition with Use of Digital Images. Nutrients, 11, (7), 1-15. DOI: $10.3390 /$ nu11071627

Poussel, M., Laure, P., Genest, J., Fronzaroli, E., Renaud, P., Favre, A., Chenuel, B. (2014). Sleep and academic performance in young elite athletes. Archives of Pediatrics, 21, (7),722-726. DOI: 10.1016/j.arcped.2014.04.016

Taylor, L., Chrismas, B., Dascombe, B., Chamari, K. \& Fowler, P. (2016). Sleep Medication and Athletic Performance-The Evidence for Practitioners and Future Research Directions. Frontiers in Physiology, 7, 83,1-5. DOI: 10.3389/fphys.2016.00083

Trakman, G., Forsyth, A., Hoye, R. \& Belski, R. (2019). Australian team sports athletes prefer dietitians, the internet and nutritionists for sports nutrition information. Nutrition \& Dietetics, 76, 4, 369-372. DOI: 10.1111/1747-0080.12569

Urdampilleta, A., Martínez, J., Julia, S. \& Álvarez, J. (2013). Protocolo de hidratación antes, durante y después de la actividad físico-deportiva. Motricidad. European Journal of Human 
Movement, 31, 57-76. Disponible en: https://www.redalyc.org/pdf/2742/27422958600 4.pdf 